\title{
Use of Vitamin D With Anti-Tumor Necrosis Factor Therapy for Crohn's Disease
}

\author{
Mauro Bafutto a, b, d, Enio Chaves Oliveira ${ }^{\text {a, c }}$, Joffre Rezende Filho ${ }^{\text {a, b }}$
}

\begin{abstract}
Background: Vitamin D (VD) has an important role in regulating gut mucosal immunity, and seems to be inversely linked to disease activity and more frequent relapses in inflammatory bowel disease. In this study, we evaluated patients with Crohn's disease (CD) treated with anti-tumor necrosis factor (TNF) in association with VD.
\end{abstract}

Methods: A double-blind, randomized, prospective study was conducted. Thirty patients with a history of moderate to severe $\mathrm{CD}$, in use of anti-TNF, of both sexes, 18 to 70 years, with the dosage of VD $<$ $75 \mathrm{nmol} / \mathrm{L}(30 \mathrm{ng} / \mathrm{mL})$ were randomized and divided into three groups: group 1 (G1): 10 patients received 2,000 IU VD, per os (PO)/week for 8 weeks; group 2 (G2): 10 patients received 10,000 IU VD, PO/week for 8 weeks; group 3 (G3): 10 patients received 50,000 IU VD, PO/week for 8 weeks. Before and at the end of 8 weeks patients were submitted to VD, fecal calprotectin (FC) and C-reactive protein (CRP) dosage. Follow-up period was 52 weeks, and they are checked for disease activity recurrence (Crohn's disease activity index (CDAI) > 150, FC > 300 and computerized tomography (CT) scan), FC, CRP, and VD levels.

Results: Increased VD levels were observed in all groups $(\mathrm{P}<$ $0.0001)$. CRP did not change. There was a significant decrease of FC in $\mathrm{G} 3(1,014 \pm 850$ vs. $483 \pm 564 ; \mathrm{P}=0.04)$, no significant decrease in G2 $(76,767 \pm 751$ vs. $535 \pm 823 ; \mathrm{P}=0.2)$ and increase in $\mathrm{G} 1(1,101 \pm$ 744 vs. $1,357 \pm 819 ; \mathrm{P}=0.4$ ). During the 52 -week follow-up period, it was showed that recurrent disease activity $(\mathrm{CDAI}>150, \mathrm{FC}>200$ and $\mathrm{CT}$ scan) was predominant in patients with $\mathrm{VD}<30$ group, and the remission rate was predominant in patients with $\mathrm{VD}>30$ group $(\mathrm{P}=0.0001)$. A statistically significant difference in VD levels was noted in $\mathrm{CD}$ patients after 52 weeks that presented flare or disease remission $(\mathrm{P}=0.001)$.

Conclusions: Use of VD associated with anti-TNF treatment may im-

Manuscript submitted April 21, 2020, accepted May 14, 2020

Published online June 18, 2020

anstituto Goiano de Gastroenterolgoia, Goiania, Brazil

bDepartment of Internal Medicine, School of Medicine, Universidade Federal de Goias, Goiania, Brazil

${ }^{\mathrm{c}}$ Department of Surgery, School of Medicine, Universidade Federal de Goias, Goiania, Brazil

${ }^{\mathrm{d} C o r r e s p o n d i n g ~ A u t h o r: ~ M a u r o ~ B a f u t t o, ~ I n s t i t u t o ~ G o i a n o ~ d e ~ G a s t r o e n t e r o l-~}$ goia, Goiania, BrazilGoiania, Brazil. Email: maurobafutto@yahoo.com.br

doi: https://doi.org/10.14740/gr1264 prove clinical response in $\mathrm{CD}$. VD levels greater than $30 \mathrm{ng} / \mathrm{mL}$ have better rates of remission.

Keywords: Vitamin D; Crohn's disease; Anti-TNF-alpha therapy; Biologic therapy; Inflammatory bowel disease

\section{Introduction}

Inflammatory bowel disease (IBD) that includes ulcerative colitis (UC) and Crohn's disease (CD) is a chronic relapsing-remitting and progressive inflammatory disease of unclear etiology. It is thought to result from a complex interaction between genetic predisposition, a dysregulated immune response, and an environmental trigger.

It is recognized that the availability of vitamin $\mathrm{D}(\mathrm{VD})$ is essential in regulating gut mucosal immunity, and their studies suggest that VD may affect epithelial integrity of digestive tube, innate immunological barrier function, and the development and function of T cells. IBD patients frequently evolve with VD deficiency with prevalence being higher than the general population and somewhat higher in CD than UC [1-3].

VD deficiency in IBD seems to be inversely linked to disease activity, more frequent relapses, higher rates of postoperative recurrence, poorer quality of life, and in general, an abnormal response to biologicals as compared with patients with normal or high serum 25 -hydroxyvitamin D (S-25(OH) D) levels [4]. In this study, we used different doses of VD in patients with $\mathrm{CD}$ that have been treated with anti-tumor necrosis factor (TNF) and observed the clinical and laboratory outcome.

\section{Materials and Methods}

The Ethics Committee of the Hospital Alberto Rassi approved the study in clinical research (CEPHGG protocol number 783/15). This study was conducted in compliance with the ethical standards of the responsible institution on human subjects as well as with the Helsinki Declaration. All patients signed an informed consent form to participate in the study. The patients were recruited on Instituto Goiano de Gastroenterologia, Goiania, Goias, Brazil.

This was a double-blind, randomized, prospective study. Forty-two patients with a history of moderate to severe CD, 


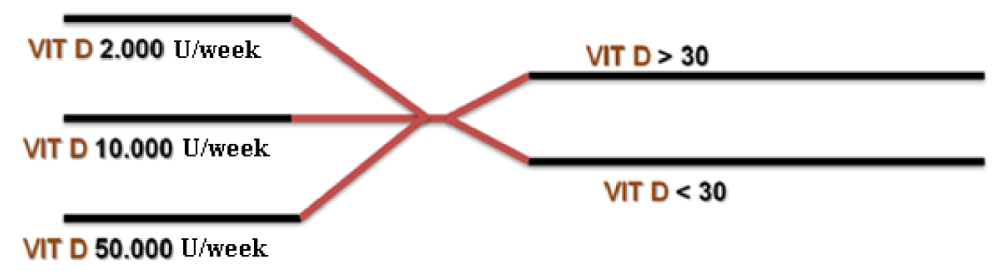

I. Intervention - 8 weeks

II. Observation - 52 weeks

Figure 1. Study design of vitamin D dosage and groups at the intervention period of 8 weeks and the observation period of 52 weeks.

in use of anti-TNF, of both sexes, between 18 to 70 years, with the dosage of $\mathrm{VD}<75 \mathrm{nmol} / \mathrm{L}(30 \mathrm{ng} / \mathrm{mL})$, nonsmokers, who signed the informed consent, were selected. Exclusion criteria include: patients less than 18 or over 70 years of age, pregnant women, those with chronic kidney or liver disease, sarcoidosis, tuberculosis, hyper- or hypoparathyroidism, neoplasia, use of anticonvulsants, and patients who received calcium supplements or VD in the last 6 months. Thirty patients were randomized. Patients were submitted to a questionnaire of sun exposure, quality of life (Inflammatory Bowel Disease Questionnaire (IBDQ)), clinical examination, VD dosage, C-reactive protein (CRP), and fecal calprotectin (FC).

The patients were divided into three groups: group 1 (G1): 10 patients received 2,000 IU VD, per os (PO)/week for 8 weeks; group 2 (G2): 10 patients received 10,000 IU VD, $\mathrm{PO} /$ week for 8 weeks; and group $3(\mathrm{G} 3)$ : 10 patients received 50,000 IU VD, PO/week for 8 weeks.

At the end of 8 weeks, the patients answered the IBDQ and were submitted to VD, FC and CRP dosage.

Disease activity surveillance was based on clinical laboratory and radiologic parameters: Crohn's disease activity index $(\mathrm{CDAI})>150$ for clinical disease activity; FC $>300$ for laboratory sign of disease activity; and computerized tomography (CT) scan for signals of disease activity.

At each visit, the patients were submitted to CDAI and FC. The patients with abnormal results of CDAI and FC were submitted to CT scan. All patients were followed for 52 weeks and checked for disease activity recurrence (CDAI $>150, \mathrm{FC}$ $>300$, CT scan), FC, CRP, and VD levels (Fig. 1).

\section{Statistics}

Continuous variables were compared using the Mann-Whitney test and the Student's $t$-test, as appropriate. Categorical variables were assessed using Fisher's exact test. The P value < 0.05 was considered statistically significant. All analyses were performed using the GraphPad ${ }^{\circledR}$ 8.1.2 software.

\section{Results}

Thirty CD patients (Table 1) in anti-TNF treatment and low VD were screened. The mean age was 37 years, and the rela- tion of male/female was 1:1. Most patients had CD beginning between 17 and 40 years old. The location of the disease was mainly ileocolonic and ileal, respectively, and structuring behavior was predominant. The duration of CD was determined as less than 5 years in $53 \%$ of patients, and $47 \%$ had more than 5 years of the disease.

After randomization, three groups were performed to the intervention period as follow: G1 group has 10 patients (eight females and two males, age (M): 41 years); G2 group has 10 patients (eight males and two females, age (M): 37 years); G3 group has 10 patients (five males and five females), age (M): 33 years).

Clinical data before starting VD supplement are shown in

Table 1. Demographic Data of Patients $(N=30)$

\begin{tabular}{|c|c|}
\hline Variables & Results \\
\hline Age (mean) (years) & 37 \\
\hline \multicolumn{2}{|l|}{ Gender } \\
\hline Male & 15 \\
\hline Female & 15 \\
\hline \multicolumn{2}{|l|}{ Age of diagnosis (Montreal classification) } \\
\hline A1 (below 16 years) & 2 \\
\hline A2 (between 17 and 40 years) & 23 \\
\hline A3 (above 40 years) & 5 \\
\hline \multicolumn{2}{|l|}{ Location } \\
\hline L1 (ileal) & 11 \\
\hline L2 (colonic) & 4 \\
\hline L3 (ileocolonic) & 15 \\
\hline \multicolumn{2}{|l|}{ Behavior } \\
\hline B1 (non-stricturing, non-penetrating) & 8 \\
\hline B2 (stricturing) & 10 \\
\hline B3 (penetrating) & 7 \\
\hline $\mathrm{B} 2+\mathrm{B} 3$ & 5 \\
\hline Perianal disease & 5 \\
\hline \multicolumn{2}{|l|}{ Duration of disease } \\
\hline$<5$ years & 16 \\
\hline$>5$ years & 14 \\
\hline Basal vitamin D & 19.3 \\
\hline
\end{tabular}


Table 2. Patients Data Before Starting Vitamin D Supplement

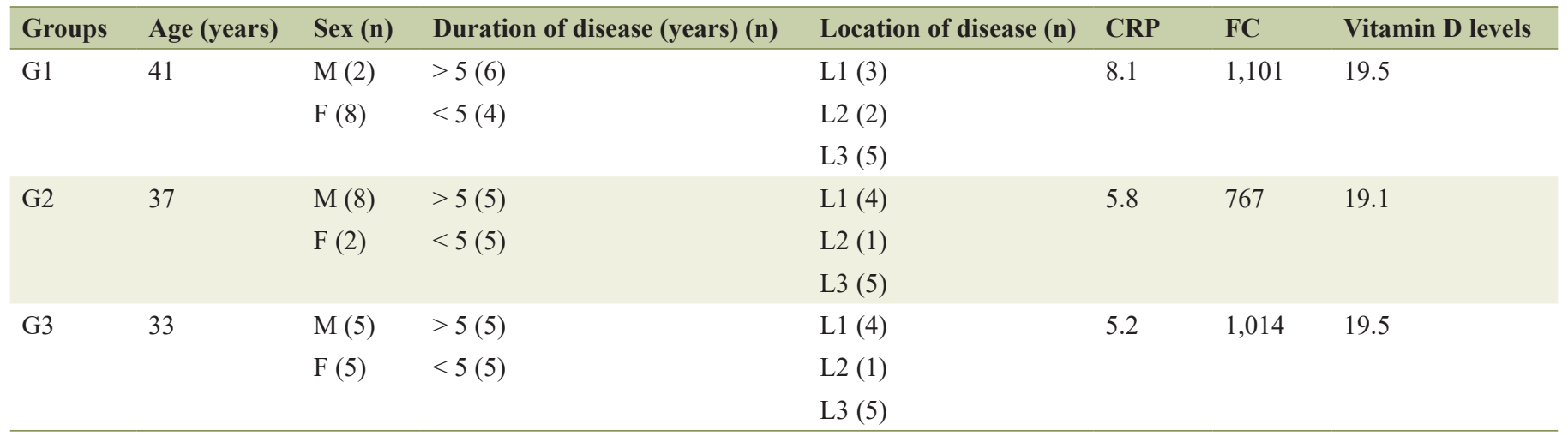

M: male; F: female; CRP: C-reactive protein; FC: fecal calprotectin; L1: ileal; L2: colonic; L3: ileocolonic.

Table 2.

IBDQ improvement was observed in all groups with statistically significant results in $\mathrm{G} 2(\mathrm{P}=0.04)$ and $\mathrm{G} 3(\mathrm{P}=0.01)$.

Increased VD levels were observed in all groups (mean \pm standard deviation $(\mathrm{SD})$ vs. mean $\pm \mathrm{SD})$ : G1 $(19.5 \pm 5.1$ vs. $26 \pm 6.7 ; \mathrm{P}=0.07) ; \mathrm{G} 2(19.1 \pm 4.1$ vs. $26 \pm 5.8 ; \mathrm{P}=0.04) ; \mathrm{G} 3$ $(19.5 \pm 6.4$ vs. $46.4 \pm 12.7 ; \mathrm{P}<0.0001)$ (Fig. 2$)$.

CRP dosage were reduced, although not statistically significant, in $\mathrm{G} 2(5.8 \pm 4$ vs. $3.9 \pm 2.8 ; \mathrm{P}=0.18)$ and $\mathrm{G} 3(5.2 \pm$ 7.3 vs. $2.4 \pm 3.6 ; \mathrm{P}=0.2)$; and increased in $\mathrm{G} 1(8.1 \pm 10.3$ vs. $13.4 \pm 19.9 ; \mathrm{P}=0.3$ ) (Fig. 3).
There was a significant decrease of FC in G3 $(1,014 \pm 850$ vs. $483 \pm 564 ; \mathrm{P}=0.04)$, no significant decrease in $\mathrm{G} 2(767 \pm$ 751 vs. $535 \pm 823 ; \mathrm{P}=0.2)$, and increase in $\mathrm{G} 1(1,101 \pm 744$ vs. $1,357 \pm 819 ; \mathrm{P}=0.4$ ) (Fig. 4 ).

After the intervention period of 8 weeks two groups were performed for observation period as follow: VD $<30$ group (18 patients: 10 female cases and eight male); and VD $>30$ group (12 patients: five female cases and seven male).

After 52-week follow-up period, it was showed that recurrent disease activity (CDAI $>150, \mathrm{FC}>200$ and $\mathrm{CT}$ scan) was predominant in patients with $\mathrm{VD}<30$ group and the re-

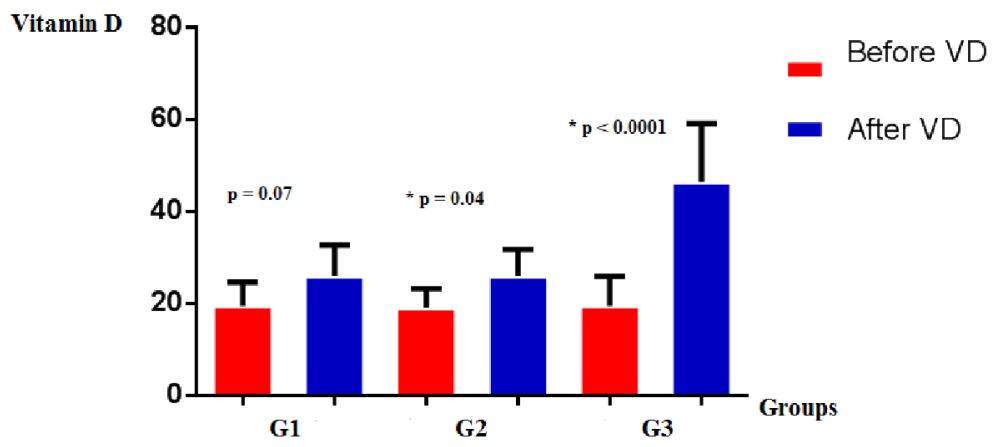

Figure 2. Vitamin D levels in groups $\mathrm{G} 1, \mathrm{G} 2$, and $\mathrm{G} 3$, before and after supplementation.

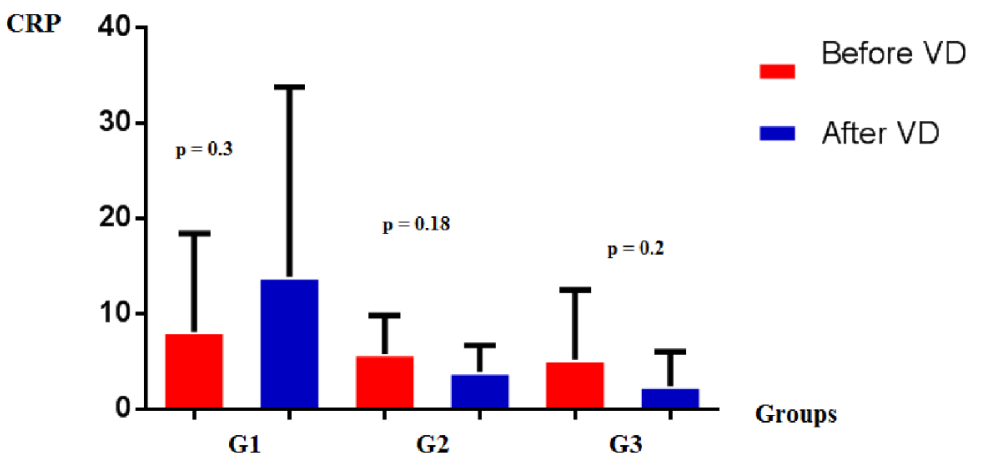

Figure 3. CRP levels in groups G1, G2, and G3, before and after vitamin D supplementation. CRP: C-reactive protein. 


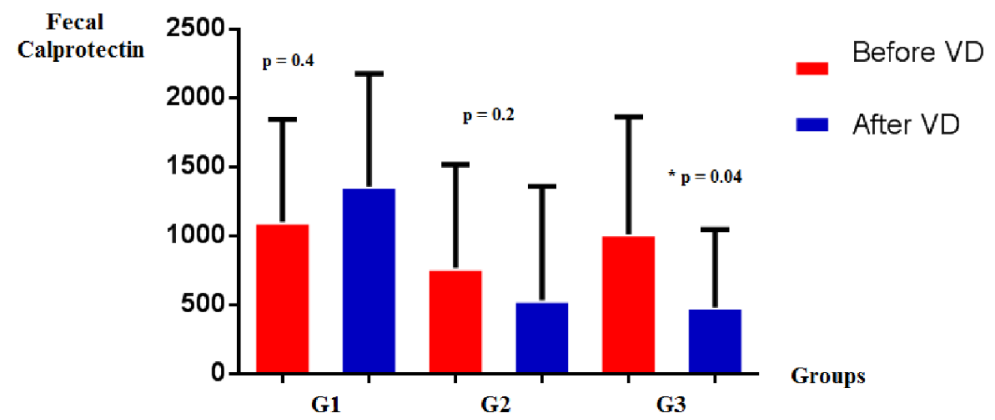

Figure 4. FC levels in groups G1, G2, and G3, before and after vitamin D supplementation. FC: fecal calprotectin.

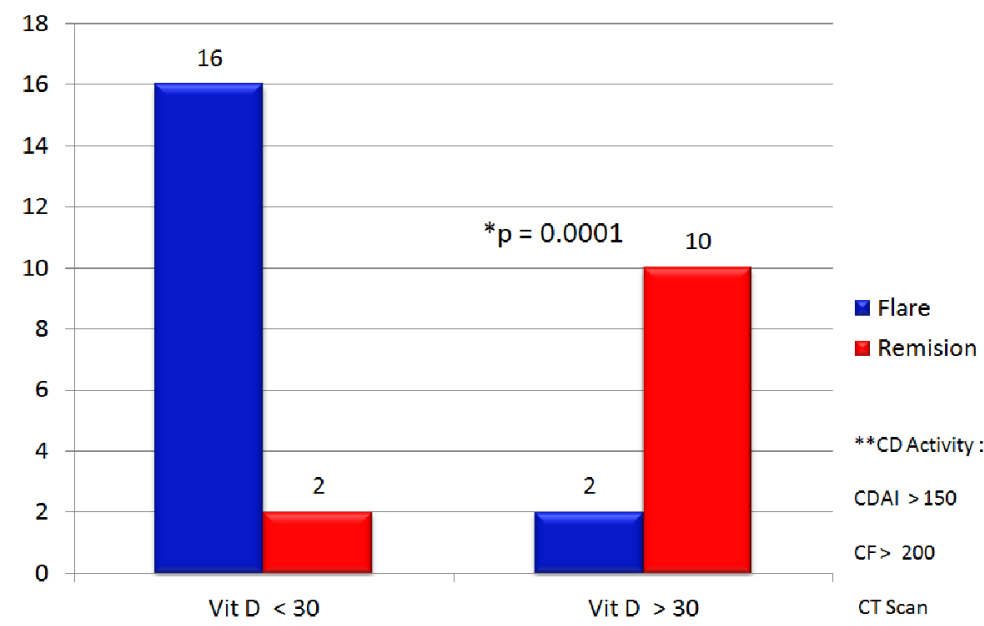

Figure 5. Patients with $C D$ activity in the observation period, during 52 weeks follow-up with vitamin $D>30$ or $<30$. ${ }^{* *} C D$ activity: CDAI > 150, FC > 200 and CT scan. CD: Crohn's disease; FC: fecal calprotectin; CDAl: Crohn's disease activity index; CT: computerized tomography.

mission rate was predominant in patients with $\mathrm{VD}>30$ group $(\mathrm{P}=0.0001)$ (Fig. 5). Also statistical significant results were observed in FC $(\mathrm{P}=0.02)$ and $\mathrm{CRP}(\mathrm{P}=0.01)$ when compared patients with $\mathrm{VD}>30$ and $\mathrm{VD}<30$.

A statistically significant difference in VD levels was noted in CD patients after an observation period of 52 weeks that presented flare or disease remission (mean \pm SD vs. mean \pm SD): $(42.6 \pm 15.5$ vs. $21.2 \pm 6.0 ; \mathrm{P}=0.001)$ (Fig. 6).

\section{Discussion}

VD seems to be important to immunologic response with the maintenance of intestinal epithelium, modulation of inflammatory of the innate and immune response, and tolerogenic adaptive immune response [5]. Gastrointestinal microbiota is linked to the barrier function of VD. Serum VD is being correlated with changes in gastrointestinal microbiome associated with inflammatory immune responses [6].

Many studies have evaluated the prevalence of VD deficiency and insufficiency in people with IBD. VD deficiency is generally higher in patients with CD than UC and usually higher than that of the general population [1].
VD deficiency in IBD is associated with increased disease activity, cancer risk and anemia. Insufficient VD level at initiation of anti-TNF-alpha treatment were more likely to stop treatment due to loss of response (hazard ratio (HR): 3.49;

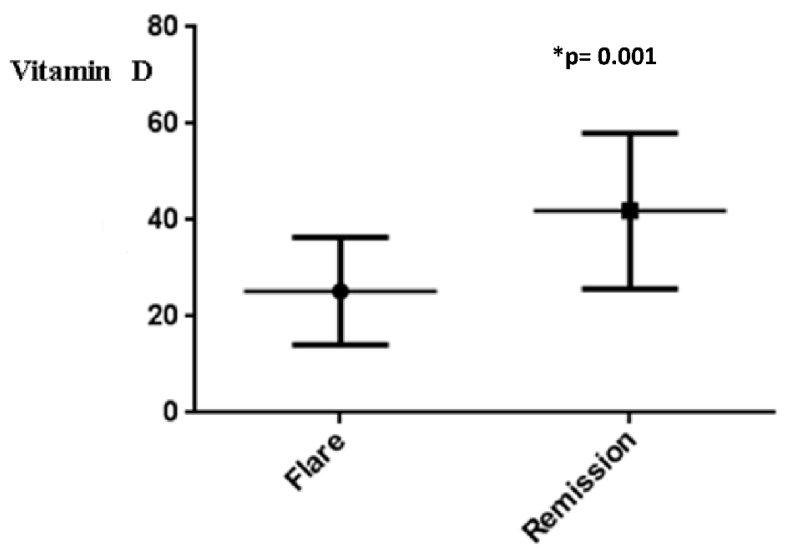

Figure 6. Levels of vitamin $D$ in $C D$ patients after an observation period of 52 weeks that presented flare or disease remission. CD: Crohn's disease. 
95\% confidence interval (CI): 1.34 - 9.09) [1].

There is a lot of clinical evidence about the role of VD therapy in IBD. It is inversely linked to disease activity [7]; oral VD is safe with few side effects and preventing bone deterioration [8], reduction of respiratory infections [9], decreases hospitalization and the need for surgery [10]. A VD analog was shown to act as a TNF-alpha inhibitor of peripheral blood mononuclear cells [11].

In the present study, we used VD at different doses in combination with anti-TNF for CD patients for 8 weeks. We observed the clinical and laboratory response for 52 weeks. We noted the improvement of VD levels and laboratory biomarkers at the end of 8 weeks mainly at the G3 group that received $50,000 \mathrm{UI} /$ week. At the observation period of 52 weeks, we noted statistically significant difference at VD levels between patients in remission or presented flare and that CD activity (CDAI $>150, \mathrm{FC}>200$, and CT scan) was reduced in patients that presented VD levels above $30 \mathrm{ng} / \mathrm{mL}$.

The results of the present study confirm recent publications that related VD with anti-TNF therapy. It was showed that VD treatment inhibits the TNF pathway and suppresses IBD [12], enhances the ability of anti-TNF therapy to suppress dendritic cell activity in CD [13]; VD levels may influence initial response to anti-TNF medication, low VD levels may predispose patients to decreased odds of remission [14], and that VD levels may influence durability of anti-TNF induction and maintenance therapy [15].

There were some limitations in our study, mainly regarding a small number of patients and the absence of a placebo control group. However, the study had some strengths, such as comparing three different doses of VD, observation period for 52 weeks, and results regarding VD treatment with clinical and laboratory improvement in both the intervention and observation periods.

As previously described, the immunologic effects of VD, the combination therapy with anti-TNF used in this study presented a good clinical and laboratory outcome. In this way, the results suggest that VD may have a synergistic effect with anti-TNF therapy in CD patients.

In conclusion, the use of VD associated with anti-TNF treatment is related to improve clinical and laboratory response in CD patients; and VD levels greater than 30 may provide higher rates of $\mathrm{CD}$ remission.

\section{Acknowledgments}

None to declare.

\section{Financial Disclosure}

None to declare.

\section{Conflict of Interest}

All authors have no conflict of interest to declare.

\section{Informed Consent}

Informed consents were obtained from the study participants.

\section{Author Contributions}

MB contributed to project development, data collection and manuscript editing; ECO and JRF contributed to data analysis and manuscript writing.

\section{Data Availability}

The authors declare that data supporting the findings of this study are available within the article.

\section{References}

1. Fletcher J, Cooper SC, Ghosh S, Hewison M. The role of vitamin $\mathrm{D}$ in inflammatory bowel disease: mechanism to management. Nutrients. 2019;11(5).

2. Bakshi A, Nguyen H, Borum M. Vitamin D screening by gastroenterologists in patients with inflammatory bowel disease. Am J Gastroenterol. 2009;104(3):791.

3. Raftery T, Martineau AR, Greiller CL, Ghosh S, McNamara D, Bennett K, Meddings J, et al. Effects of vitamin D supplementation on intestinal permeability, cathelicidin and disease markers in Crohn's disease: Results from a randomised double-blind placebo-controlled study. United European Gastroenterol J. 2015;3(3):294-302.

4. Nielsen OH, Rejnmark L, Moss AC. Role of Vitamin D in the Natural History of Inflammatory Bowel Disease. J Crohns Colitis. 2018;12(6):742-752.

5. Garg M, Lubel JS, Sparrow MP, Holt SG, Gibson PR. Review article: vitamin D and inflammatory bowel disease-established concepts and future directions. Aliment Pharmacol Ther. 2012;36(4):324-344.

6. Luthold RV, Fernandes GR, Franco-de-Moraes AC, Folchetti LG, Ferreira SR. Gut microbiota interactions with the immunomodulatory role of vitamin D in normal individuals. Metabolism. 2017;69:76-86.

7. Sadeghian M, Saneei P, Siassi F, Esmaillzadeh A. Vitamin D status in relation to Crohn's disease: Meta-analysis of observational studies. Nutrition. 2016;32(5):505-514.

8. Hlavaty T, Krajcovicova A, Payer J. Vitamin D therapy in inflammatory bowel diseases: who, in what form, and how much? J Crohns Colitis. 2015;9(2):198-209.

9. Arihiro S, Nakashima A, Matsuoka M, Suto S, Uchiyama K, Kato T, Mitobe J, et al. Randomized trial of vitamin D supplementation to prevent seasonal influenza and upper respiratory infection in patients with inflammatory bowel disease. Inflamm Bowel Dis. 2019;25(6):10881095.

10. Ananthakrishnan AN, Cagan A, Gainer VS, Cai T, Cheng SC, Savova G, Chen P, et al. Normalization of plasma 25-hydroxy vitamin $\mathrm{D}$ is associated with reduced risk 
of surgery in Crohn's disease. Inflamm Bowel Dis. 2013;19(9):1921-1927.

11. Stio M, Treves C, Martinesi M, d'Albasio G, Bagnoli $\mathrm{S}$, Bonanomi AG. Effect of anti-TNF therapy and vitamin D derivatives on the proliferation of peripheral blood mononuclear cells in Crohn's disease. Dig Dis Sci. 2004;49(2):328-335.

12. Zhu Y, Mahon BD, Froicu M, Cantorna MT. Calcium and 1a,25-dihydroxy vitamin D3 target the TNF-pathway to suppress experimental inflammatory bowel disease. Eur J Immunol. 2005;35:217-224.

13. Hendy P, Reddi D, Barnardo D, Durant RL. Vitamin D enhances the ability of anti-TNF therapy to suppress den- dritic cell activity in Crohn's disease. Gut.2016;65(Suppl 1): A148.2-A149.

14. Winter RW, Collins E, Cao B, Carrellas M, Crowell AM, Korzenik JR. Higher 25-hydroxyvitamin D levels are associated with greater odds of remission with anti-tumour necrosis factor-alpha medications among patients with inflammatory bowel diseases. Aliment Pharmacol Ther. 2017;45(5):653-659.

15. Zator ZA, Cantu SM, Konijeti GG, Nguyen DD, Sauk J, Yajnik V, Ananthakrishnan AN. Pretreatment 25-hydroxyvitamin D levels and durability of anti-tumor necrosis factor-alpha therapy in inflammatory bowel diseases. JPEN J Parenter Enteral Nutr. 2014;38(3):385-391. 